MATEC Web of Conferences 44, 01038 (2016)

DOI: $10.1051 /$ matecconf/20164401038

(c) Owned by the authors, published by EDP Sciences, 2016

\title{
The Key Solution Algorithm of Linear Programming Model
}

\author{
Jun Liu ${ }^{1, a}$, Chuan Cheng Zhao ${ }^{1}$, Zhi Guo Ren ${ }^{1}$, Zhong Yi Feng ${ }^{1}$, Zheng Ping Zhu ${ }^{1}$ \\ 1. Lanzhou City University, School of Information Science and engineering, Lanzhou 730070, P.R. China
}

\begin{abstract}
Linear programming problem is a common problem, and to solve the linear model is more plagued. The paper generating algorithm is based on mathematical theory and composition. The design of feasible solution algorithm illustrates key linear programming model, then we can find a better way to solve the linear programming model solutions.
\end{abstract}

\section{Introduction}

According to mathematical theory of linear programming model $^{[1-2]}(1)$ :

$\max \quad z=\sum_{i=1}^{n} c_{i} x_{i}$

s.t $\left\{\begin{array}{c}\sum_{j=1}^{n} a_{i j} x_{j} \leq a_{i n+1}(i=1,2, \cdots, m) \\ x_{i} \geq 0(i=1,2, \cdots, n)\end{array}(m \geq n)\right.$

$S, t$ condition of the equation

$$
\sum_{j=1}^{n} a_{i j} x_{j}=a_{i n+1}(i=1,2, \cdots, m)
$$

And $x_{i}=0(i=1,2, \cdots, n) m+n$ equations form an optimal solution $m+n$ plane (side) closed set $\Omega$, the linear programming model (1) on the closed set $\Omega$ border on the objective function $\mathrm{s}, \mathrm{t}$ condition of the equation:

$$
z=\sum_{i=1}^{n} c_{i} x_{i}
$$

From $(0,0, \cdots, 0)(z=0)$ moving along the surface normal direction, so that the value of $z$ is gradually increased until the value of the maximum, then getting a linear programming model (1) of the optimal solution $\left(x_{1}, x_{2}, \cdots, x_{n}\right)$ and the corresponding $z$ maximum. This method is theoretically established, but in practice it would be inconvenient to solve over three-dimensional linear programming model ${ }^{[3]}$.
This paper designs the key feasible solution algorithmsof computer of graphic linear programming model.

\section{The Combination Generation Algorithm}

In the key feasible solution algorithms of computer of graphic linear programming model, it is supposed to discuss the combination generation algorithm of $C_{n}^{m}, C_{n}^{m}$ linear equations are generated from $\mathrm{m}+\mathrm{n}$ equations ${ }^{[4]}$.

Let $C_{n}^{0}=\varnothing, C_{n}^{n}=1,2,3, \cdots, n$. When generating the combination of $C_{n}^{m}, n$ numbers of the generated combination, each and all combinations are arranged by the dictionary order.

\subsection{The Generation of Combination $C_{n}^{1}$}

The combination of $C_{1}^{1}$ is $1, C_{2}^{1}$ is $1,2, C_{3}^{1}$ is $1,2,3 \ldots$, and the combination of $C_{n}^{1}$ is $1,2,3, \ldots, \mathrm{n}$. Combination can be expressed as follows (to be expressed simply, written $C_{n}^{m}$ as $\left.\Delta(n, m)\right)$, borrowing documents ${ }^{[2]}$ to discuss the digital triangles and trigonometric operations and " $\oplus$ " and the triangular numbers counted by "。"):

$$
\begin{aligned}
& \Delta(1,1)=(1)^{T}, \\
& \Delta(2,1)=\left(\begin{array}{lll}
1 & 2
\end{array}\right)^{T}, \\
& \Delta(3,1)=\left(\begin{array}{lll}
1 & 2 & 3
\end{array}\right)^{T}, \\
& \ldots, \\
& \Delta(n, 1)=\left(\begin{array}{llllll}
1 & 2 & 3 & \cdots & n-1 & n
\end{array}\right)^{T}
\end{aligned}
$$

authore-mail: 527876625@.qq.com

This studywas supported by Lanzhou City University Ph.D. Research Fund (21265099,41361013,GS[2013]GHB1084,

LZCU-BS2013-09 and LZCU-BS2013-12). 
(To write conveniently, matrix form is used above.)

\subsection{The Generation of Combination $C_{n}^{2}$}

The combination of $C_{2}^{2}$ is $12, C_{3}^{2}$ is $12,13,23, \ldots, C_{n}^{2}$ is $12,13,14, \ldots, 1 n ; 23,24, \ldots 2 n ; \ldots . . ; n-2 n-1, n-1 n$; $n-1 n$.

It can be expressed by digital triangle as follows: $\Delta(2,2)=12=\Delta(1,2) \oplus \Delta(1,1) \circ 2$,

$$
\begin{aligned}
& \Delta(3,2)=\begin{array}{ll}
12 & 13 \\
23
\end{array}=\Delta(2,2) \oplus \Delta(2,1) \circ 3 \\
& \begin{array}{lll}
12 & 13 & 14
\end{array} \\
& \Delta(4,2)=\quad 23 \quad 24=\Delta(3,2) \oplus \Delta(3,1) \circ 4 \\
& 34 \\
& \begin{array}{llllll}
12 & 13 & 14 & \ldots & 1 n-1 & 1 n
\end{array} \\
& \begin{array}{lllll}
23 & 24 & \cdots & 2 n-1 & 2 n
\end{array} \\
& \Delta(n, 2)= \\
& 34 \quad \ldots \quad 3 n-1 \quad 3 n \\
& (n-2)(n-1) \quad(n-2) n \\
& (n-1) n
\end{aligned}
$$

$=\Delta(n-1,2) \oplus \Delta(n-1,1) \circ n$

\subsection{The Generation of Combination $C_{n}^{3}$}

Similarly, itcan get the digital triangle indicating the combinations of $C_{3}^{3}, C_{4}^{3}, C_{5}^{3}, \ldots, C_{n}^{3}$.

$$
\begin{aligned}
& \Delta(3,3)=123=\Delta(2,3) \oplus \Delta(2,2) \circ 3 \\
& \Delta(4,3)=\begin{array}{llllll}
123 & 124 & 134 \\
& & 234
\end{array}=\Delta(3,3) \oplus \Delta(3,2) \circ 4 \\
& \Delta(5,3)= \\
& \begin{array}{llllll}
123 & 124 & 134 & 125 & 135 & 145 \\
& & 234 & 235 & 245 \\
& & & & 345
\end{array}
\end{aligned}
$$$$
=\Delta(4,3) \oplus \Delta(4,2) \circ 5
$$$$
\ldots,
$$$$
\Delta(n, 3)=\begin{array}{lllllll}
123 & 124 & 134 & 125 & 135 & 145 & \cdots \\
& 234 & & & 235 & 245 & \cdots \\
& & & & & 345 & \cdots
\end{array}
$$

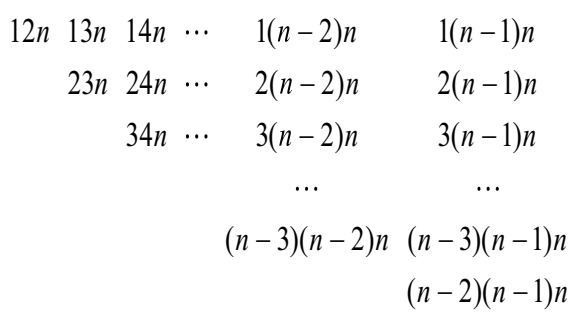$$
=\Delta(n-1,3) \oplus \Delta(n-1,2) \circ n
$$

\subsection{The Generation of Combination $\Delta(n, m)\left(C_{n}^{m}\right)$}

Similarly, itcan obtain the combination of $\Delta(n, m)$.

Thus, itcan get the $\Delta(n, m)$ of combination $C_{n}^{m}$ generating algorithm theorem.

Theorem: the combination of $C_{n}^{m}(m \leq n / 2, m \geq 2)$ is

$$
\Delta(n, m)=\Delta(n-1, m) \oplus \Delta(n-1, m-1) \circ n .
$$

\section{The Design of Key Feasible Solution Algorithm}

From the foregoing, if the feasible solutions of linear programming model (1) is the closed set $\Omega$, and the optimal solution is in the boundary of this closed set $\Omega$, and the possible case is unique (a vertex of closed set $\Omega$ ) or infinite (an edge or a surface of closed set $\Omega$ ). So it only need to find each vertex of the closed set $\Omega$ when calculating. These vertices of the closed set $\Omega\left(x_{1}, x_{2}, \cdots, x_{n}\right)$ are called key feasible solutions, then determining the optimal solution of the linear programming model (1) from key feasible solution ${ }^{[5-10]}$.

To find the key feasible solution on the closed set $\Omega$, changing the restrictions of linear programming model to:

$$
\left\{\begin{array}{c}
\sum_{j=1}^{n} a_{i j} x_{j}=a_{i n+1}(i=1,2, \cdots, m) . \\
x_{i}=0(i=1,2, \cdots, n)
\end{array} .\right.
$$

This is a linear equation containing $n$ variables and $\mathrm{m}+\mathrm{n}$ equations, the key feasible solution of the closed set $\Omega$ is the intersection from $\mathrm{m}+\mathrm{n}$ equations obtained $\mathrm{C}_{\mathrm{m}+\mathrm{n}}^{\mathrm{n}}$ equations. The intersection satisfies the points of restrictions in model (1),and it corresponds to the value of the objective function $z$. Finding the maximum value from these values, there may be three cases, one (unique), two (an edge) or three (a surface).

Computer algorithm as following:

(1) Let $\max =0$;

(2) Generating $m+n$ equations to $C_{m+n}^{n}$ equation groups by the algorithm of generated combination and solving it;

(3) Substituting the linear programming model (1) restrictions, judging whether it is the key feasible solution on the closed set $\Omega$, if so, calculating the value of objective function $\mathrm{z}$;

(4) Judging whether $z>\max$ is established, if so, $\max =\mathrm{z}$ returningthe key feasible solution of equation groups is $\left(x_{1}, x_{2}, \cdots, x_{n}\right)$. If $z=\max$ is satisfied, keeping the key feasible solution of equation groups as $\left(x_{1}, x_{2}, \cdots, x_{n}\right)$;

(5) Returning to 1, until getting the solution of $C_{m+n}^{n}$ equation groups;

(6) Outputting the value of $\mathrm{z}$ and the optimal solution $\left(x_{1}, x_{2}, \cdots, x_{n}\right)$

With this algorithm, we can easily calculate the optimal solution of linear programming model (1) $\left(x_{1}, x_{2}, \cdots, x_{n}\right)$

\section{The Advantages of Algorithm}

This algorithm is based on the principle of the 
existence of the optimal solution on the closed set $\Omega$, directly calculating the intersection and determining whether it is the key feasible solution of the closed set $\Omega$, and immediately seeking $z$, greatly reducing the number of calculations, and occupying less memory as a fast and effective way. Meanwhile, it will have a good reference for the algorithm of integer programming by improving this algorithm. This section will be discussed about the pull-off algorithm of the solutions of integer programming model in another text.

\section{References}

1. Prof. Dr. T. Gal. Homogene mehrparametrische lineare Programmierung[J]. Zeitschrift für Operations Research . 1972 (3)

2. K. -H. Borgwardt. The Average number of pivot steps required by the Simplex-Method is polynomial[J]. Zeitschriftfür Operations Research . 1982 (1)

3. Wolfgang Temelt. The parameter space of the general linear programming problem[J]. Soviet Mathematical Journal . 1967 (3)

4. The ellipsoid method and its consequences in combinatorial optimization[J]. Combinatorica . 1981 (2)

5. N. Karmarkar. A new polynomial-time algorithm for linear programming[J]. Combinatorica . 1984 (4)

6. Yamamura K,TanakaS.Performance evaluation of the LPtest algorithm for finding all solutions of piecewise-linearresistive circuits. International Journal of CircuitTheory and Applications . 2000

7. H. P. Benson,D. Lee. Outcome-based algorithm for optimizing over the efficient set of a bicriteria linear programming problem[J]. Journal of Optimization Theory and Applications . 1996 (1)

8. F. P. Vasil'ev,M. Yachimovich. Discrepancy method for the lexicographic linear programming problem[J]. Computational Mathematics and Modeling . 1995 (1)

9. J. Fülöp,L. D. Muu. Branch-and-Bound Variant of an Outcome-Based Algorithm for Optimizing over the Efficient Set of a Bicriteria Linear Programming Problem[J]. Journal of Optimization Theory and Applications . 2000 (1)

10. Adi Ben-Israel. A $\mathbb{Z}$-Simplex Algorithm with partial updates[J]. BIT . 1987 (1)

\section{$6 \mathrm{C}$ language source code}

\#include $<$ stdio.h $>$ \#include $<$ stdlib.h $>$ \#include $<$ string.h $>$ \#include $<$ math.h $>$ \#define N 100

\#define OK 1

\#define ERROR 0

double a $[\mathrm{N}][\mathrm{N}], \mathrm{c}[\mathrm{N}][\mathrm{N}]$;

typedefstruct

\{

double number[N];

doublereasult;

\}

SeqList;

typedefstruct

\{

\section{SeqListrecord[N];}

\}Node;

intNsize,Size;

int Print(double b[][N],intn,int m)

\{

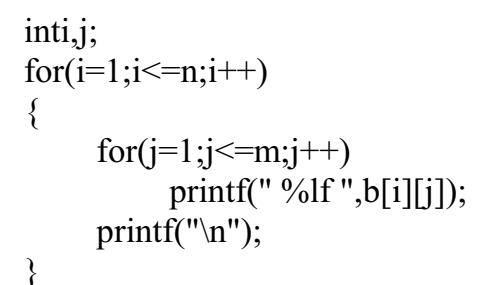

return $\mathrm{OK}$;

\}

void Print1(Node *L)

\{

inti,j;

for $(\mathrm{i}=1 ; \mathrm{i}<=\mathrm{L}->$ Size $; \mathrm{i}++)$

for $(\mathrm{j}=1 ; \mathrm{j}<=\mathrm{L}->$ Nsize $; \mathrm{j}++)$

\{

printf("\n");

$\operatorname{printf}\left(" \mathrm{i}=\% \mathrm{~d} \quad \% \mathrm{lf}^{\prime}, \mathrm{i}, \mathrm{L}->\operatorname{record}[\mathrm{i}]\right.$. number[j]]);

\}

printf("\n");

void Print $2($ Node $*$ L)

\{

inti,j;

for $(\mathrm{i}=1 ; \mathrm{i}<\mathrm{L}->$ Size $; \mathrm{i}++)$

\{

for $(\mathrm{j}=1 ; \mathrm{j}<=\mathrm{L}->$ Nsize $; \mathrm{j}++)$ printf(" \%e ",L->record[i].number[j]); printf("\n"); printf(" The sult is:\%lf ",L->record[i].reasult)

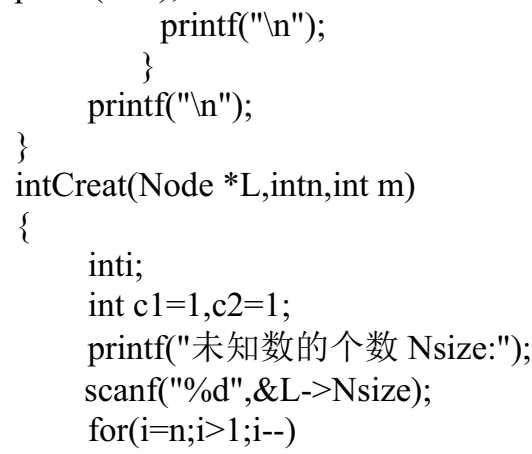

inti;

int $\mathrm{c} 1=1, \mathrm{c} 2=1$; printf("未知数的个数 Nsize:"); scanf("\%d",\&L->Nsize); for $(\mathrm{i}=\mathrm{n} ; \mathrm{i}>1 ; \mathrm{i}--)$ 


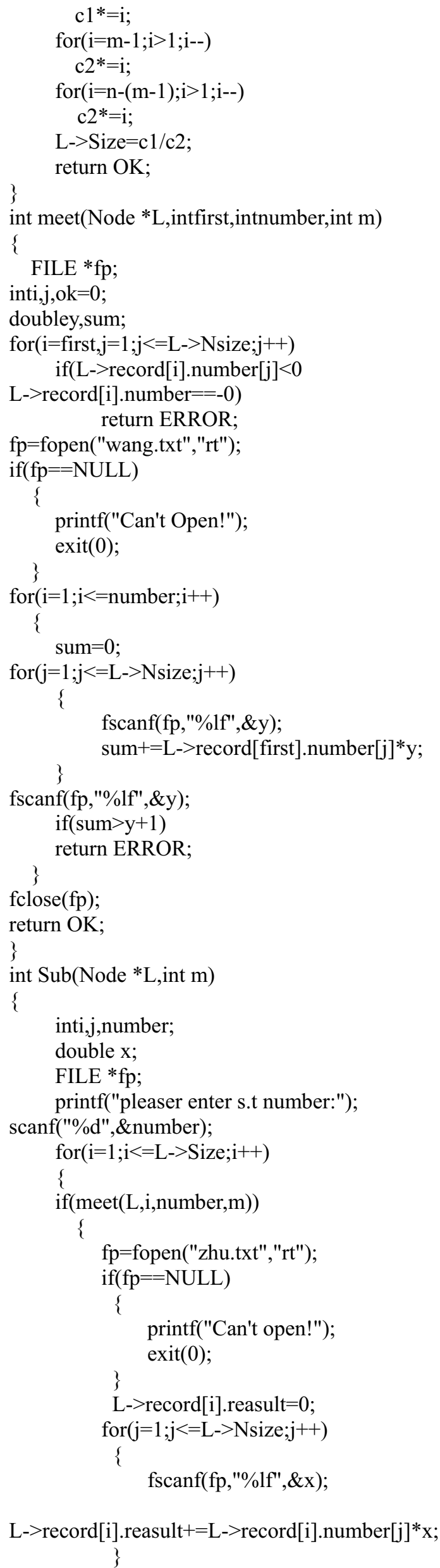

fclose(fp);

else L->record[i].reasult $=0$;

\}

return $\mathrm{OK}$;

int Memory(double b[][N],Node *L,int m)

\{

int $\mathrm{j}$;

staticinti $=1$;

for $(\mathrm{j}=1 ; \mathrm{j}<=\mathrm{L}->\mathrm{Nsize} ; \mathrm{j}++)$

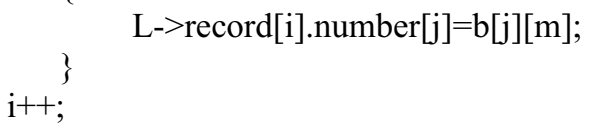


return $\mathrm{OK}$;

\}

intuptriangle(double $\mathrm{b}[][\mathrm{N}]$,Node ${ }^{*}$ L,intn,int m)

\{

inti,j,k;

double s;

for $(\mathrm{i}=1 ; \mathrm{i}<=\mathrm{n} ; \mathrm{i}++)$

\{

change(b,i,n,m);

for $(j=i+1 ; j<=n ; j++)$

\{

$\mathrm{s}=\mathrm{b}[\mathrm{j}][\mathrm{i}] / \mathrm{b}[\mathrm{i}][\mathrm{i}]$;

if $(\mathrm{s}==0)$ break;

for $(\mathrm{k}=1 ; \mathrm{k}<=\mathrm{m} ; \mathrm{k}++)$

\{ \}

$\mathrm{b}[\mathrm{j}][\mathrm{k}]=(-1 * \mathrm{~s}) * \mathrm{~b}[\mathrm{i}][\mathrm{k}]+\mathrm{b}[\mathrm{j}][\mathrm{k}]$;

\}

Tackback(b,L,n,m);

return $\mathrm{OK}$;

\}

int Assemble(double b[][N],Node *L,intn,int m)

\{

inti,j,k,elem[100];

$\mathrm{i}=1 ; \operatorname{elem}[\mathrm{i}]=1$;

while $(\mathrm{i} !=0)$

\{

if $($ elem $[\mathrm{i}]>\mathrm{n})$

\{

$$
\text { i--; }
$$

elem $[\mathrm{i}]++$;

\}

else if $(\mathrm{i}==\mathrm{m}-1)$

\{

for $(\mathrm{j}=1 ; \mathrm{j}<=\mathrm{m}-1 ; \mathrm{j}++)$

for $(\mathrm{k}=1 ; \mathrm{k}<=\mathrm{m} ; \mathrm{k}++)$

$\mathrm{c}[\mathrm{j}][\mathrm{k}]=\mathrm{b}[\mathrm{elem}[\mathrm{j}]][\mathrm{k}]$; uptriangle $(\mathrm{c}, \mathrm{L}, \mathrm{m}-1, \mathrm{~m})$;

memset(c,0,sizeof(c)); elem[i]++;

\}

else

\{

$\mathrm{i}++$;

elem $[\mathrm{i}]=\operatorname{elem}[\mathrm{i}-1]+1$; \}

\}

return $\mathrm{OK}$;

\}

int main()

\{

FILE *fp;

inti,j,m,n;

Node list;

printf("pleaser enter $\mathrm{n}$ and $\mathrm{m}: ")$;

scanf("\%d\%d",\&n,\&m);

fp=fopen("wang.txt","rt");

if $(\mathrm{fp}==\mathrm{NULL})$

\{

printf("Can't open!");

exit(0);

for $(\mathrm{i}=1 ; \mathrm{i}<=\mathrm{n} ; \mathrm{i}++)$

for $(j=1 ; j<=m ; j++)$

\{

fscanf(fp,"\%lf",\&a[i][j]); \}

creat(\&list,n,m);

assemble(a,\&list,n,m);

$\operatorname{sub}(\&$ list, $m$ );

print2(\&list);

return 0 ;

\} 